\title{
Minimally Invasive Management of Diverticular Disease
}

\author{
Andrea Madiedo, MD ${ }^{1}$ Jason Hall, MD, MPH, FACS, FACRS ${ }^{1}$ \\ ${ }^{1}$ Department of Surgery, Boston Medical Center, Boston, \\ Massachusetts \\ Address for correspondence Jason F. Hall, MD, FGH Building, Room \\ \#5017, 820 Harrison Avenue, Boston, MA 02118 \\ Clin Colon Rectal Surg 2021;34:113-120. \\ (e-mail: Jason.Hall@bmc.org).
}

\begin{abstract}
Keywords

- complicated diverticulitis

- minimally invasive sigmoidectomy

- laparoscopic sigmoidectomy

- robotic sigmoidectomy

- inferior mesenteric artery (IMA) ligation

- splenic flexure mobilization

- ICG immunofluorescence

Traditionally, management of complicated diverticular disease has involved open damage control operations with large definitive resections and colostomies. Studies are now showing that in a subset of patients who would typically have undergone an open Hartmann's procedure for Hinchey III/IV diverticulitis, a laparoscopic approach is equally safe, and has better outcomes. Similar patients may be good candidates for primary anastomosis to avoid the morbidity and subsequent reversal of a colostomy. While most operations for diverticulitis across the country are still performed open, there has been an incremental shift in practice toward minimally invasive approaches in the elective setting. The most recent data from large trials, most notably the SIGMA trial, found laparoscopic sigmoid colectomy is associated with fewer short-term and long-term complications, decreased pain, improvement in length of stay, and maintains better cost-effectiveness than open resections. Some studies even demonstrate that robotic sigmoid resections can maintain a similar if not more reduction in morbidity as the laparoscopic approach while still remaining cost-effective.

Intraoperative approaches also factor into improving outcomes. One of the most feared complications in colorectal surgery is anastomotic leak, and many studies have sought to find ways to minimize this risk. Factors to consider to minimize incidence of leak are the creation of tension-free anastomoses, amount of contamination, adequacy of blood supply, and a patient's use of steroids. Techniques supported by data that decrease anastomotic leaks include preoperative oral antibiotic and mechanical bowel prep, intraoperative splenic flexure mobilization, low-tie ligation of the inferior mesenteric artery, and use of indocyanine green immunofluorescence to assess perfusion.

In summary, the management of benign diverticular disease is shifting from open, morbid operations for a very common disease to a minimally invasive approach. In this article, we review those approaches shown to have better outcomes, greater patient satisfaction, and fewer complications.
\end{abstract}

The role of operative therapy in patients with diverticulitis is to address the burden of complicated or recurrent disease. Although traditional approaches call for open damage control operations and large definitive resections, there has been a slow shift in practice toward less-invasive approaches to the management of complicated diverticular disease. With the ongoing technological and biomedical advances in the field of surgery, there are an increasing number of minimally invasive approaches available that can improve patient outcomes. Minimally invasive approaches employ the same principles
Issue Theme Diverticulitis; Guest Editor: Jason Hall, MD, MPH, FACS, FACRS (c) 2021. Thieme. All rights reserved. Thieme Medical Publishers, Inc., 333 Seventh Avenue, 18th Floor, New York, NY 10001, USA
DOI https://doi.org/ 10.1055/s-0040-1716703. ISSN 1531-0043. 
of sound open operative management; source control and resection of the affected colon, attention to preservation of nearby structures without injury, and re-establishment of bowel continuity and functionality continue to be important facets of care.

\section{Preoperative Considerations}

\section{Endoscopic Stenting}

Diverticular strictures occur as a result of chronic inflammation, scarring, and fibrosis from repeated bouts of diverticulitis. While many patients may not present with complete large bowel obstruction, obstructive symptoms or progressive constipation should trigger further evaluation by colonoscopy for underlying malignancy. In some cases, endoscopic stenting has been employed to palliate strictures. Some retrospective series have found that stents are less effective and are associated with more complications such as reobstruction, migration, and perforation when used in the management of benign diseases such as diverticulitis. ${ }^{1}$ Although stents can be used as a bridge to operative management, surgeons should be aware of the potential complications and be facile with surgical resection as the definitive form of management. ${ }^{2}$

\section{Preoperative Bowel Preparation}

The role of preoperative bowel preparation has been extensively studied in colorectal surgery. It has become clear that the addition of preoperative oral antibiotics in addition to parenteral antibiotics and mechanical bowel preparation is superior in reducing surgical site infections, and may play a role in decreasing readmission rates, ${ }^{3}$ anastomotic leaks as well as the duration of postoperative ileus. ${ }^{3,4}$ There have been no large randomized control trials looking at different combinations of bowel preparation versus no prep. However, recent American College of Surgeons National Surgical Quality Improvement Program (ACS-NSQIP) studies have shown superiority of combined preoperative mechanical and oral antibiotic bowel preparations. ${ }^{5}$ The limitation to these studies is the use of large retrospective databases and small sample size of patients undergoing antibiotic prep alone. Use of oral antibiotic bowel prep alone has shown inconsistent outcomes with regard to SSI and anastomotic leak across multiple studies but it does provide an argument for antibiotic bowel prep alone in patients who are at higher risk of dehydration, kidney injury, or too frail to tolerate mechanical bowel preparation. ${ }^{4,6}$

\section{Ureteral Stenting}

Ureteral injury during colon surgery has been found to range anywhere from $<1 \%$ to as high as $7 \%$. Ureteral stenting can be considered in cases where a difficult dissection is anticipated secondary to malignancy, pelvic radiation, or other inflammatory conditions. A large retrospective NSQIP-based study found prophylactic ureteral catheter placement to be associated with lower risk of ureteral injury. ${ }^{7}$ Other proponents for ureteral catheterization have reported experiences of complete prevention of injury with the use of lighted ureteral catheters, which provide an additional visual cue in the

\section{Hinchey Classification}

Stage I Pericolic abscess or phlegmon

Stage II Pelvic, intra-abdominal or retroperitoneal abscess

Stage III Generalized purulent peritonitis

Stage IV Generalized fecal peritonitis

Modified Hinchey Classification

Stage 0 Mild clinical diverticulitis

Stage Ia Confined pericolic inflammation/phlegmon

Stage Ib Confined pericolic abscess

Stage II Pelvic, distant intraabdominal or retroperitoneal

abscess

Stage III Generalized purulent peritonitis

Stage IV Fecal peritonitis

Fig. 1 Hinchey and modified Hinchey classification. (Fischer JE. Mastery of Surgery. Philadelphia: Wolters Kluwer; 2019.)

absence of tactile feedback in laparoscopy. ${ }^{8,9}$ This is still new technology that is being developed and is not widely popular yet. While stents are not necessary nor do they definitively decrease the incidence of ureteral injury, stenting may facilitate detection of injury at the time of operation. One must balance this against the potential risks of complications from these stents. Reports have noted urinary tract infections, hematuria, ureteral damage from catheter placement, urinary retention from ureteral edema, longer operative time, length of stay, readmission, and increase costs with placement of these catheters. ${ }^{9}, 10$

\section{Operative Approaches}

Operative approaches are largely based on patient's Hinchey classification (-Fig. 1), patient's anatomy and disease process, as well as surgeon training and level of comfort with each approach. While the traditional approach to colectomies for diverticular disease had been open, laparoscopic and robot-assisted colectomies are becoming increasingly common. This is especially true in the elective setting due to improved postoperative outcomes. ${ }^{11}$

\section{Emergent Sigmoidectomy for Diverticular Disease}

Open sigmoidectomy has traditionally been the operative management of choice in peritonitis from Hinchey 3 or Hinchey 4 diverticulitis, or in the setting of relative inexperience with a laparoscopic approach. The steps and approach to sigmoidectomy are similar laparoscopically as open. The affected sigmoid colon is exposed to identify the area of active or recurrent disease, and proximal and distal resection sites are identified. A medial to lateral approach involves identification and ligation of the inferior mesenteric artery (IMA) and further dissection inferior to the mesentery along the edge of the colon, taking care to lift these structures while pushing down the gonadal vessels, ureters, and Gerota's fascia. ${ }^{12}$ A lateral to medial approach involves taking down the colon at the line of Toldt and working medially to lift the colon off the retroperitoneum. This approach sometimes necessary if there is extensive scarring and foreshortening of the mesentery from prior phlegmons limiting the 
surgeon's ability to take a medial to lateral approach. The medial to lateral or lateral to medial approaches are dictated by surgeon preference and anatomy.

A few points are important to note regarding resection margins. Distally, the goal is to resect the entirety of the sigmoid colon down to the splaying of the tinea onto the rectum as the distal resection margin is commonly the site of recurrence of diverticulitis if the sigmoid is not fully resected. ${ }^{13}$ The exception to this is if one is performing a Hartmann's procedure and there is uninvolved sigmoid distally that may be spared until the time of reversal. Proximally, it is not required to excise all diverticulum-containing colon. It is, however, important to note and resect the areas involved in acute or chronic inflammation. Finally, if there is concern for cancer in a segment of diverticulitis and the patient has not had a colonoscopy to evaluate for malignancy, a cancer operation must be performed such as a formal left hemicolectomy, not limited to a sigmoidectomy.

The resection margins and extent of disease guide the dissection that can be approached in various ways.

\section{Restoration of Continuity}

Restoration of continuity (Hartmann's vs primary anastomosis) in patients who are taken to the operating room emergently for source control of Hinchey class III or IV complicated diverticulitis is a topic of current investigation and debate. There is data to suggest that ostomies from a Hartmann's are not reversed at a rate of up to $40 \% .^{14,15}$ The first step in approaching the patient with purulent or feculent peritonitis is to assess the degree of contamination and inflammation upon entering the abdomen to help guide ultimate decision for source control and diversion versus definitive resection in the same trip to the operating room. While some patients may be too hemodynamically unstable or unfit to undergo primary anastomosis, there is a cohort of patients who can avoid the morbidity of a Hartmann's procedure without difference in outcomes. ${ }^{16}$ A large multicenter randomized controlled trial from France studied the outcomes of patients undergoing emergent operation for Hinchey III and IV diverticulitis and found no mortality benefit, but did show an advantage in ostomy reversal rates in patients undergoing primary anastomosis. ${ }^{17}$

Recent data aims to validate a minimally invasive approach to the management of purulent and feculent peritonitis (Hinchey III and IV) in the appropriate patient setting. ${ }^{18,19}$ The traditional method has been to approach these patients using a conventional laparotomy, however there is now data from a meta-analysis and systematic review by Cirocchi et al to support superior postoperative outcomes, shorter length of stay, and higher Hartmann reversal rates in patients who have undergonelaparoscopic sigmoid resection. This is in contrast to other retrospective NSQIP-based studies that fail to find a significant advantage of laparoscopy over open in the emergent setting. ${ }^{20}$ Laparoscopic washout on the other hand is not superior to sigmoid resection for Hinchey III and IV diverticulitis as patients have no significant improvement in outcomes and greater morbidity with abscess formation requiring percutaneous drainage and additional procedures including operative resection. ${ }^{21-23}$ This approach is discussed in another article in this issue.

\section{Elective Sigmoidectomy for Diverticular Disease}

\section{Laparoscopic}

In the setting of elective sigmoidectomy for diverticular disease, a minimally invasive laparoscopic approach is the recommended operative management. ${ }^{24}$ This is based on the SIGMA trial, a large multicenter double-blind randomized control trial in Europe to examine outcomes between laparoscopic and open sigmoid resection for diverticulitis. ${ }^{25}$ Shortterm results of the trial showed a $15.4 \%$ significantly decreased incidence of major postoperative complications, as well as decreased postoperative pain, reported quality of life and hospital length of stay compared with open sigmoid resection. ${ }^{26}$ Further analyses of patients 6 months out from their operation continue to show decreased late complications as well in the laparoscopic group to include development of hernias, adhesive small bowel obstruction, anastomotic stricture, enterocutaneous fistulas, and recurrence of their disease. $^{27}$ This finding has been confirmed by other studies. ${ }^{28,29}$

Some authors have proposed that laparoscopic sigmoid resection is associated with longer operative times and larger costs with no other postoperative differences. ${ }^{30,31}$ However, costs for patients undergoing laparoscopic and open colectomy enrolled in the SIGMA trial were comparable. ${ }^{32}$ Other studies, such as one out of the Cleveland Clinic by Senagore et al, and a nationwide administrative database study of over 18,000 patients have also found decreased costs associated with laparoscopic sigmoid colectomies. ${ }^{33}$ The difference in increased intraoperative costs is at least balanced out by the longer length of stay of patients undergoing open resection, and long-term complications. ${ }^{28-30}$

\section{Robotic}

While laparoscopic resection for symptomatic diverticulitis has favorable patient outcomes and should be the standard of care for elective resection, the most recent literature suggests that the robotic-assisted approach may result in superior outcomes. ${ }^{12,34}$ Benefits of robotic surgery include three-dimensional visualization of the field, wristed instruments for rotational range of motion in dissection of the scarred tissue and going into the pelvis, and immunofluorescence. ${ }^{12}$ A recent large national retrospective review by Raskin et al of robotic sigmoidectomy matched to open and laparoscopic controls showed significant decrease in postoperative complications rates with a robotic approach. When compared with laparoscopy, robotic surgery has a lower rate of conversion to open, postoperative ileus, and length of stay. When compared with open sigmoid resection, robotic surgery has a significantly lower rate of ileus, wound complications, renal failure, length of stay, and discharge to a rehab facility. ${ }^{12,35,36}$

Robotic surgery is associated with increased operative time and cost. Some analyses have suggested that when decreased length of stay and rates of complications are accounted for, a robotic approach is actually cost-effective compared with other procedures. ${ }^{37,38}$ Vasudevan et al found no statistical significance between total hospitalization costs of robotic colorectal surgery compared with laparoscopic approaches, estimating an average cost of $\$ 107,220$ compared with 
$\$ 114,853$, respectively. Additionally, other groups have demonstrated that operative times can be decreased over time as trained staff become accustomed to preincision preparation and docking of the robot. ${ }^{38}$

\section{Intraoperative Techniques}

\section{Splenic Flexure Mobilization}

Splenic flexure takedown is approached via dissection of the omentum off the transverse colon, staying in the infrapancreatic plane, identifying the IMV for ligation, releasing the gastrocolic and splenocolic ligaments, and extending the dissection to the lateral peritoneal fold ${ }^{39}$ (-Figs. 2-6). This allows up to $27 \mathrm{~cm}$ of colon for the creation of a tension-free colorectal anastomosis. ${ }^{40}$ Under certain circumstances, takedown of the splenic flexure during left-sided colorectal operations is necessary to achieve adequate length for a tension-free anastomosis. This is especially the case in diverticular disease as chronic inflammation and scarring limits mobilization that could lead to higher rates of postanastomotic leak, or disease recurrence.

Studies have looked at the overall rates of morbidity and mortality with selective splenic flexure mobilization and have found no significant difference. ${ }^{41}$ However, rates of iatrogenic splenic injury during splenic flexure mobilization

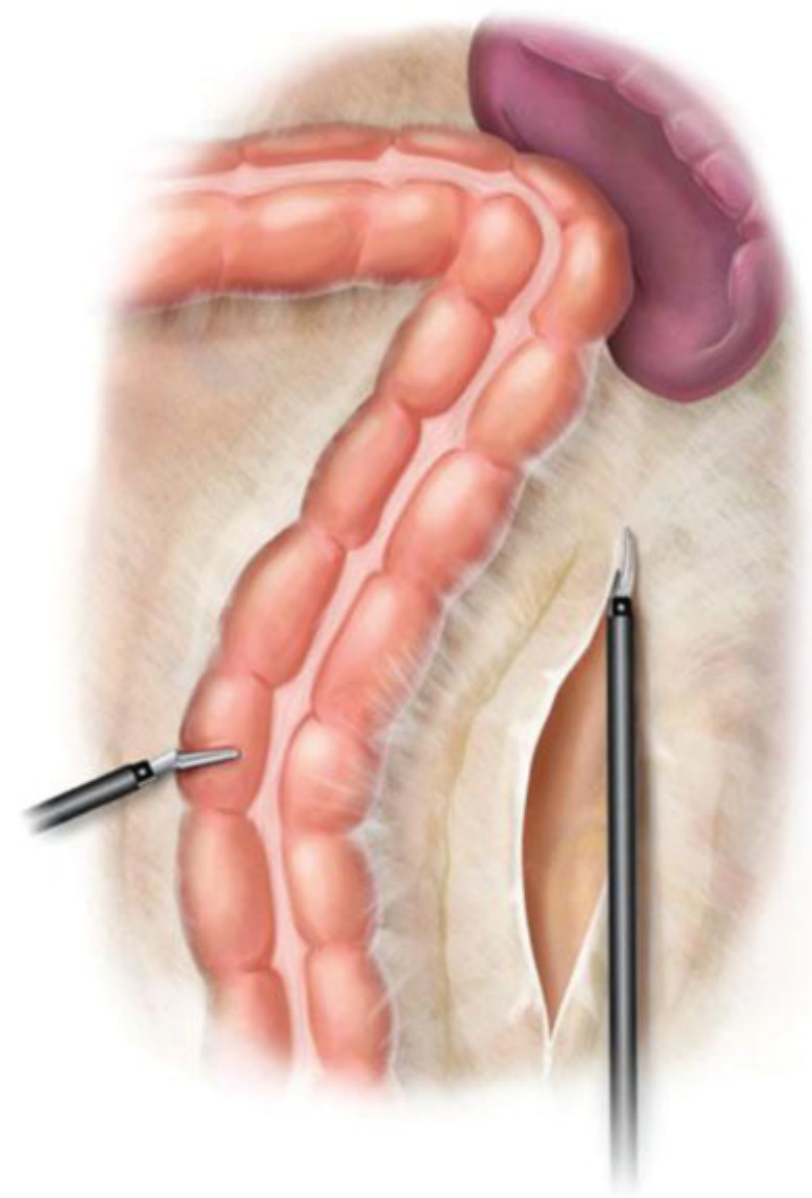

Fig. 2 Splenic flexure mobilization at the line of Toldt. (Fischer JE. Mastery of Surgery. Philadelphia: Wolters Kluwer; 2019.) have been reported up to $8 \%$, increasing patients' morbidity and risk for splenectomy. Many iatrogenic injuries occur as a result of traction and inadequate exposure. Despite lack of tactile feedback, the use of laparoscopy has been shown to reduce this risk and decrease the rate of injury, ${ }^{42}$ perhaps related to improved visualization of difficult spaces.

An additional challenge to splenic flexure mobilization is the difficulty with access and positioning in robotic surgery. While the robot is docked for optimal pelvic and left lowerquadrant dissection, it is difficult to access the adjacent field and may require laparoscopic splenic flexure takedown prior to robotic docking, ${ }^{12}$ redocking for mobilization of the splenic flexure, or development of novel techniques to access the area without redocking. ${ }^{43}$

\section{IMA Ligation Approaches}

There is no single standardized approach to the takedown of the IMA. There are two main approaches to the ligation of blood supply to the left colon in the operation for benign colon pathology. One approach involves high ligation of the IMA. This involves dissection of the IMA near its origin at the

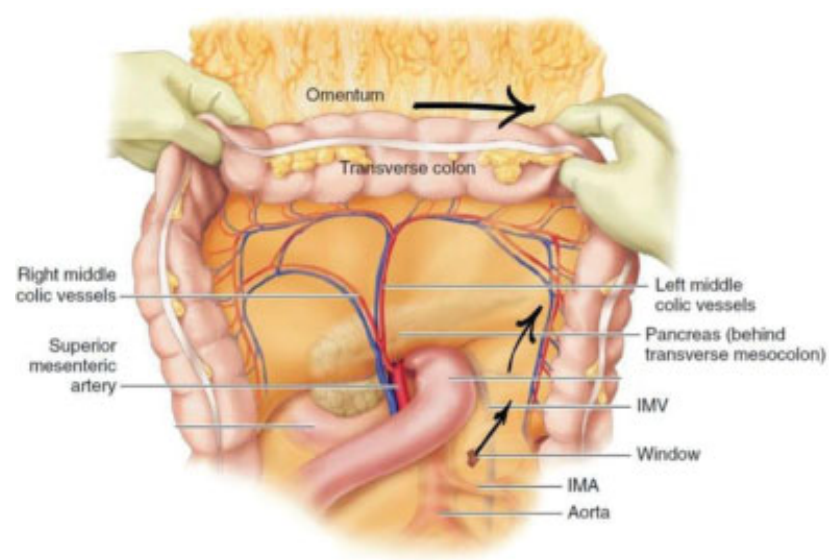

Fig. 3 Splenic flexure mobilization at the left mesocolon and omental release. (Adapted from Fischer JE. Mastery of Surgery. Philadelphia: Wolters Kluwer; 2019.) IMA, inferior mesenteric artery; IMV, inferior mesenteric vein.

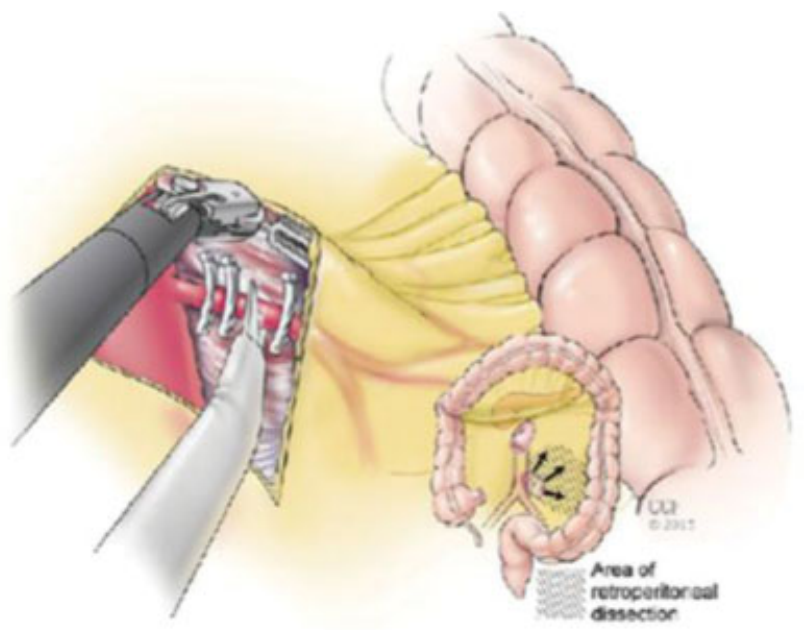

Fig. 4 Robotic entrance into the lesser sac. 


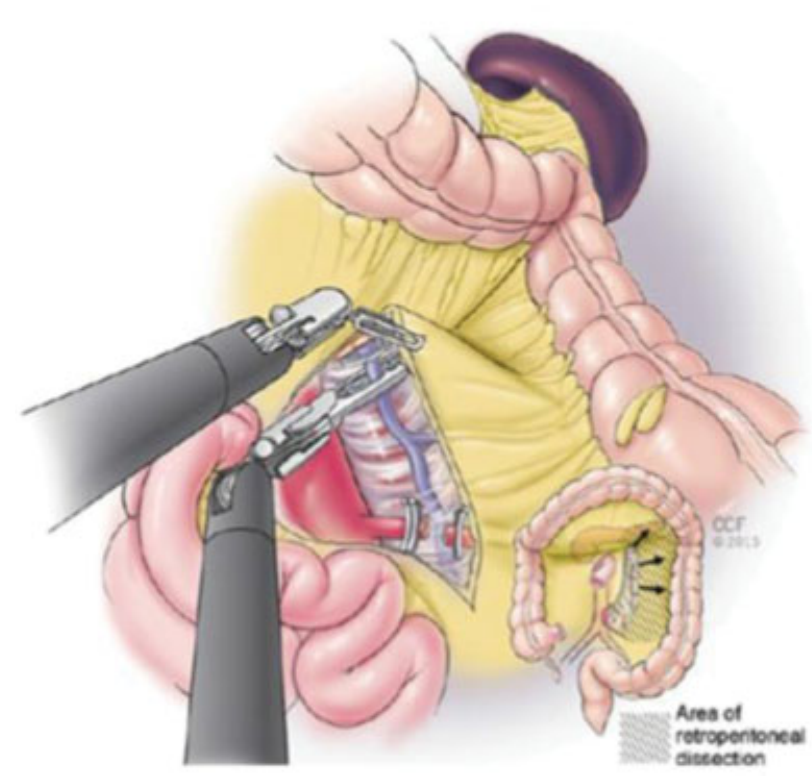

Fig. 5 Inferior mesenteric artery (IMA) ligation techniques. (A) High IMA ligation. (B) Low IMA ligation with preservation of left colic a. (C) Low IMA ligation with superior rectal artery preservation. (Adapted from Fischer JE. Mastery of Surgery. Philadelphia: Wolters Kluwer; 2019.). IMA, inferior mesenteric artery.

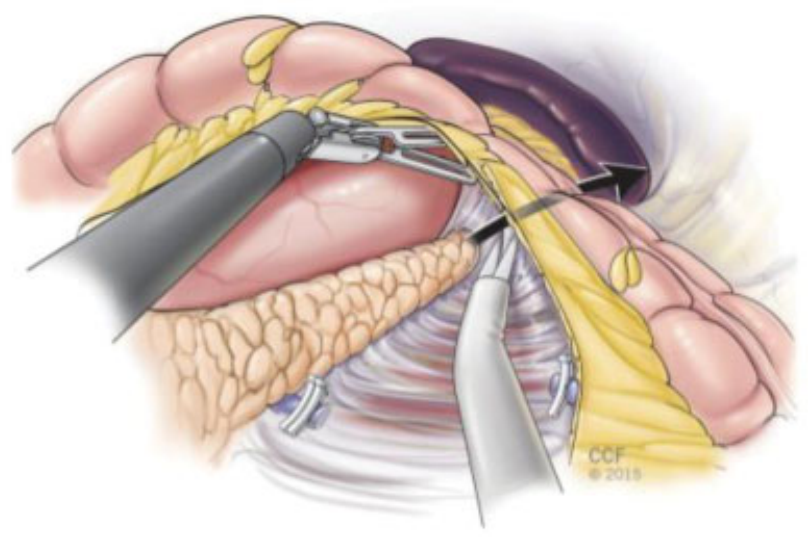

Fig. 6 Indocyanine green immunofluorescence. ${ }^{56}$ ICG, indocyanine green.

aorta and ligation. Subsequently, the entire left colon that is supplied by the IMA is taken with its mesentery up to the splenic flexure. An alternative approach involves piecemeal "low-tie IMA" ligation of perfusing branches near the wall of the colon that is to be resected ( - Fig. 7). This allows for more limited resection of the blood supply and preservation of the superior rectal artery supplying the proximal rectum.

Intraoperative factors considered in anastomotic leaks are the creation of tension-free anastomoses, amount of contamination, adequacy of blood supply, and a patient's use of steroids. Although the rectum has extensive collateralization of blood supply, there is debate whether preservation of the superior rectal artery branch off the IMA may decrease anastomotic leak rates. However, there is no strong data or well-formulated studies to date that support this theory in one direction or the other. There is one randomized control trial, by Tocchi et al, that has looked at leak rates with level of

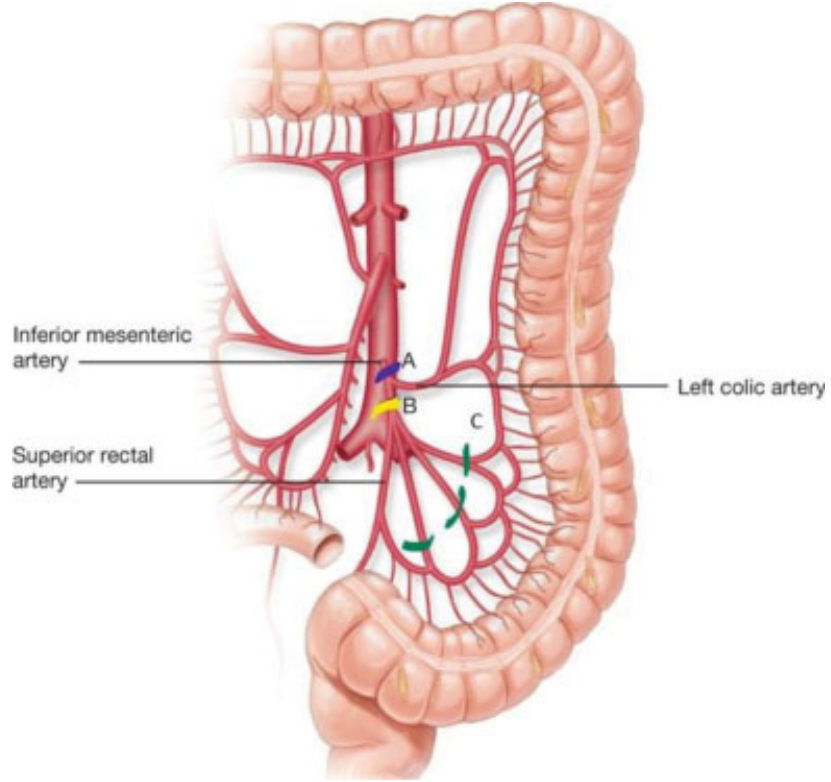

Fig. 7 Robotic inferior mesenteric artery ligation.

IMA ligation and found an increase in leaks with high IMA ligation. However, their definition of a leak included leukocytosis after day 3 and imaging evidence of change in the bowel wall thickness at the site of anastomosis. ${ }^{44}$ Other studies have failed to find this significant association with level of IMA ligation. ${ }^{45} \mathrm{~A}$ meta-analysis by Cirocchi et al attempted to compare smaller studies on this same issue but has not found a clear answer given poor data and study design most likely due to the heterogeneity in defining a colorectal anastomotic leak post-op. ${ }^{46} \mathrm{~A}$ large factor in the individual decision for where to ligate the IMA lies in the patient presentation, extensiveness of disease, and ease of dissection of the mesentery in the setting of chronic inflammatory change. Given the lack of strong data, this will remain largely a surgeon-driven decision with the knowledge that blood supply plays a large role in supporting a successful anastomosis in the appropriate patient where superior rectal artery preservation is possible.

Long-term bowel function and continence is another point to consider in the decision to preserve the IMA during sigmoidectomy for diverticular disease. To date, randomized control trials and prospective studies have sought to show that IMA preservation improves bowel function by better preservation of the sympathetic nerve plexus that runs along the IMA and the parasympathetic nerves in the pelvis. ${ }^{47,48}$ In the randomized controlled trial by Masoni et al, patients with individual sigmoid branch resection at the colonic wall with IMA preservation compared with IMA ligation distal to the origin of the left colic artery showed better colonic motility, decreased defecatory disorders, and better quality of life.

\section{Indocyanine Green Fluorescence Assessment of Bowel Perfusion}

Adequate perfusion is one of the most important factors in prevention of anastomotic leak. ${ }^{15}$ Intraoperative fluorescence imaging has grown in popularity in colorectal surgery for the 


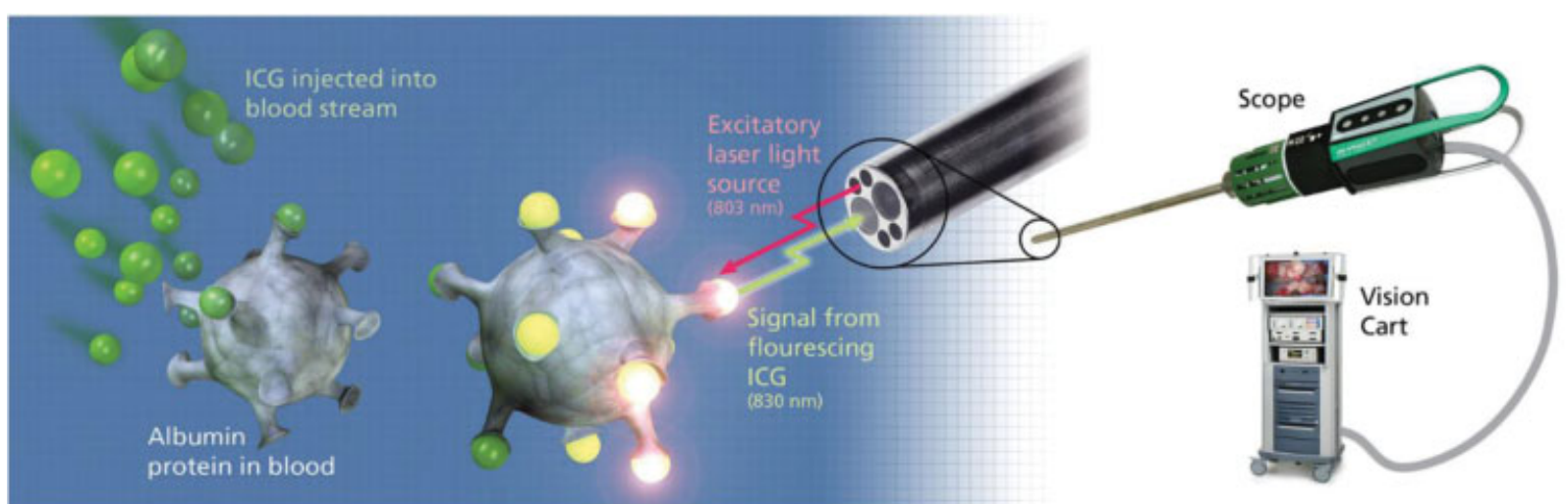

Fig. 8 Robotic ligation of the inferior mesenteric vein.

assessment of the distal anastomoses near watershed areas. Known by multiple names such as PINPOINT, IC-view, Firefly, Sky-Elite, the most commonly used fluorophore is indocyanine green (ICG) that can be injected intraarterially or intravenously at the time of anastomosis to assess the bowel viability and perfusion at the ends of planned anastomoses ${ }^{49}$ (- Fig. 8). This requires specially designed equipment to detect light emitted from the fluorophore based on degree of perfusion to the examined segment of bowel. This can be evaluated both intraabdominally during robotic and laparoscopic cases and endoscopically evaluating mucosal enhancement and perfusion with a sigmoidoscope. Multiple studies and case reports have examined the efficacy of this approach in decreasing anastomotic leak rate, the largest of which is the PILLAR II multicenter trial of 139 patients, which showed a surprising $1.4 \%$ leak rate compared with the published rate of $12 \%$ in the literature. ${ }^{50}$ Many other smaller studies have sought to also prove decreased leak rates with the use of ICG fluorescence imaging for a diverse set of disease processes such as diverticulitis, colon cancer, rectal cancer, and even esophageal resections. ${ }^{51}$ The results all reflect favorably on the use of ICG fluorescence imaging, adding only 2 to 3 minutes to the total operative time and potentially preventing a very morbid complication in a patient. The main limitation in many of these studies is the small sample size, and lack of standardization of methods to quantify adequacy of fluorescence and detect leaks. Currently, the published studies heavily rely on individual surgeons' qualitative interpretation of the fluorescence intraoperatively to determine site of anastomosis. ${ }^{51-56}$ The long-term effect of these technologies on anastomotic leak rates have yet to be determined.

\section{Summary}

There is a paradigm shift occurring in colorectal surgery toward minimally invasive approaches for the management of diverticulitis. While there is a learning curve for gaining comfort with new techniques or applying laparoscopic techniques to traditionally open approaches (especially in the case of Hinchey III and IV complicated diverticulitis), adoption of these methods is growing. The use of laparoscopy and robotic surgery has improved oncologic outcomes in colorectal surgery, and the data are now in support of its use in benign diverticular disease as well. Additionally, there is a shift in practice away from the traditional open Hartmann's procedure to a laparoscopic approach in the appropriate patient, and primary anastomosis as these factors have been shown to improve ostomy reversal rates and provide equal if not superior outcomes such as faster return of bowel function, length of stay, and ultimately costs to our healthcare system. As technology evolves, we are able to study and apply new, less-invasive techniques to a previously morbid disease process to improve patient outcomes and satisfaction.

\section{Conflict of Interest}

None declared.

\section{References}

1 Feo L, Schaffzin DM. Colonic stents: the modern treatment of colonic obstruction. Adv Ther 2011;28(02):73-86

2 Currie A, Christmas C, Aldean H, Mobasheri M, Bloom ITM. Systematic review of self-expanding stents in the management of benign colorectal obstruction. Colorectal Dis 2014;16(04):239-245

3 Anjum N, Ren J, Wang G, et al. A randomized control trial of preoperative oral antibiotics as adjunct therapy to systemic antibiotics for preventing surgical site infection in clean contaminated, contaminated, and dirty type of colorectal surgeries. Dis Colon Rectum 2017;60(12):1291-1298

4 Kiran RP, Murray ACA, Chiuzan C, Estrada D, Forde K. Combined preoperative mechanical bowel preparation with oral antibiotics significantly reduces surgical site infection, anastomotic leak, and ileus after colorectal surgery. Ann Surg 2015;262(03):416-425, discussion 423-425

5 Klinger AL, Green H, Monlezun DJ, et al. The role of bowel preparation in colorectal surgery. Ann Surg 2017. Doi: 10.1097/ SLA.0000000000002568

6 Garfinkle R, Abou-Khalil J, Morin N, et al. Is there a role for oral antibiotic preparation alone before colorectal surgery? ACSNSQIP analysis by coarsened exact matching. Dis Colon Rectum 2017;60(07):729-737

7 Coakley KM, Kasten KR, Sims SM, Prasad T, Heniford BT, Davis BR. Prophylactic ureteral catheters for colectomy: a national surgical quality improvement program-based analysis. Dis Colon Rectum 2018;61(01):84-88

8 Boyan WPJ Jr, Lavy D, Dinallo A, et al. Lighted ureteral stents in laparoscopic colorectal surgery; a five-year experience. Ann Transl Med 2017;5(03):44-44 
9 Chiu AS, Jean RA, Gorecka J, Davis KA, Pei KY. Trends of ureteral stent usage in surgery for diverticulitis. J Surg Res 2018; 222:203-211.e3

10 Leff EI, Groff W, Rubin RJ, Eisenstat TE, Salvati EP. Use of ureteral catheters in colonic and rectal surgery. Dis Colon Rectum 1982;25 (05):457-460

11 Masoomi H, Buchberg B, Nguyen B, Tung V, Stamos MJ, Mills S. Outcomes of laparoscopic versus open colectomy in elective surgery for diverticulitis. World J Surg 2011;35(09):2143-2148

12 Ragupathi M, Ramos-Valadez DI, Patel CB, Haas EM. Roboticassisted laparoscopic surgery for recurrent diverticulitis: experience in consecutive cases and a review of the literature. Surg Endosc 2011;25(01):199-206

13 Benn PL, Wolff BG, Ilstrup DM. Level of anastomosis and recurrent colonic diverticulitis. Am J Surg 1986;151(02):269-271

14 Gachabayov M, Oberkofler CE, Tuech JJ, Hahnloser D, Bergamaschi R. Resection with primary anastomosis vs nonrestorative resection for perforated diverticulitis with peritonitis: a systematic review and meta-analysis. Colorectal Dis 2018;20(09):753-770

15 Windsor A, Wexner S, Heald RJ, et al. A collaborative review of the current concepts and challenges of anastomotic leaks in colorectal surgery. Color Dis 2016;19(01):01-012

16 Tadlock MD, Karamanos E, Skiada D, et al. Emergency surgery for acute diverticulitis: which operation? A National Surgical Quality Improvement Program study. J Trauma Acute Care Surg 2013;74 (06):1385-1391, quiz 1610

17 Bridoux V, Regimbeau JM, Ouaissi M, et al. Hartmann's procedure or primary anastomosis for generalized peritonitis due to perforated diverticulitis: a prospective multicenter randomized trial (DIVERTI). J Am Coll Surg 2017;225(06):798-805

18 Cirocchi R, Fearnhead N, Vettoretto N, et al. The role of emergency laparoscopic colectomy for complicated sigmoid diverticulitis: a systematic review and meta-analysis. Surgeon 2018:1-10. Doi: 10.1016/j.surge.2018.08.010

19 Cassini D, Miccini M, Manoochehri F, Gregori M, Baldazzi G. Emergency Hartmann's procedure and its reversal: a totally laparoscopic 2-step surgery for the treatment of Hinchey III and IV diverticulitis. Surg Innov 2017;24(06):557-565

20 Turley RS, Barbas AS, Lidsky ME, Mantyh CR, Migaly J, Scarborough JE. Laparoscopic versus open Hartmann procedure for the emergency treatment of diverticulitis: a propensity-matched analysis. Dis Colon Rectum 2013;56(01):72-82

21 Vennix S, Musters GD, Mulder IM, et al; Ladies trial colloborators. Laparoscopic peritoneal lavage or sigmoidectomy for perforated diverticulitis with purulent peritonitis: a multicentre, parallel-group, randomised, open-label trial. Lancet 2015;386(10000):1269-1277

22 Schultz JK, Yaqub S, Wallon C, et al; SCANDIV Study Group. Laparoscopic lavage vs primary resection for acute perforated diverticulitis: the SCANDIV randomized clinical trial. JAMA 2015; 314(13):1364-1375

23 Shaikh FM, Stewart PM, Walsh SR, Davies RJ. Laparoscopic peritoneal lavage or surgical resection for acute perforated sigmoid diverticulitis: a systematic review and meta-analysis. Int J Surg 2017;38:130-137

24 Feingold D, Steele SR, Lee S, et al. Practice parameters for the treatment of sigmoid diverticulitis. Dis Colon Rectum 2014;57 (03):284-294

25 Klarenbeek BR, Veenhof AAFA, de Lange ESM, et al. The Sigmatrial protocol: a prospective double-blind multi-centre comparison of laparoscopic versus open elective sigmoid resection in patients with symptomatic diverticulitis. BMC Surg 2007;7:16. Doi: $10.1186 / 1471-2482-7-16$

26 Klarenbeek BR, Veenhof AA, Bergamaschi R, et al. Laparoscopic sigmoid resection for diverticulitis decreases major morbidity rates: a randomized control trial: short-term results of the Sigma Trial. Ann Surg 2009;249(01):39-44
27 Klarenbeek BR, Bergamaschi R, Veenhof AA, et al. Laparoscopic versus open sigmoid resection for diverticular disease: follow-up assessment of the randomized control Sigma trial. Surg Endosc 2011;25(04):1121-1126

28 Senagore AJ, Duepree HJ, Delaney CP, Dissanaike S, Brady KM, Fazio VW. Cost structure of laparoscopic and open sigmoid colectomy for diverticular disease: similarities and differences. Dis Colon Rectum 2002;45(04):485-490

29 Delaney CP, Chang E, Senagore AJ, Broder M. Clinical outcomes and resource utilization associated with laparoscopic and open colectomy using a large national database. Ann Surg 2008;247 (05):819-824

30 Gervaz P, Mugnier-Konrad B, Morel P, Huber O, Inan I. Laparoscopic versus open sigmoid resection for diverticulitis: long-term results of a prospective, randomized trial. Surg Endosc 2011;25 (10):3373-3378

31 Raue W, Paolucci V, Asperger W, Albrecht R, Büchler MW, Schwenk WLAPDIV-CAMIC Trial Group. Laparoscopic sigmoid resection for diverticular disease has no advantages over open approach: midterm results of a randomized controlled trial. Langenbecks Arch Surg 2011;396(07):973-980

32 Klarenbeek BR, Coupé VMH, van der Peet DL, Cuesta MA. The cost effectiveness of elective laparoscopic sigmoid resection for symptomatic diverticular disease: financial outcome of the randomized control Sigma trial. Surg Endosc 2011;25(03):776-783

33 Keller DS, Delaney CP, Hashemi L, Haas EM. A national evaluation of clinical and economic outcomes in open versus laparoscopic colorectal surgery. Surg Endosc 2016;30(10):4220-4228

34 Cassini D, Depalma N, Grieco M, Cirocchi R, Manoochehri F, Baldazzi G. Robotic pelvic dissection as surgical treatment of complicated diverticulitis in elective settings: a comparative study with fully laparoscopic procedure. Surg Endosc 2019;33 (08):2583-2590

35 Raskin ER, Keller DS, Gorrepati ML, Akiel-Fu S, Mehendale S, Cleary RK. Propensity-matched analysis of sigmoidectomies for diverticular disease. JSLS 2019;23(01):e2018.00073

36 Harr JN, Haskins IN, Amdur RL, Agarwal S, Obias V. The effect of obesity on laparoscopic and robotic-assisted colorectal surgery outcomes: an ACS-NSQIP database analysis. J Robot Surg 2018;12 (02):317-323

37 Salman M, Bell T, Martin J, Bhuva K, Grim R, Ahuja V. Use, cost, complications, and mortality of robotic versus nonrobotic general surgery procedures based on a nationwide database. Am Surg 2013;79(06):553-560

38 Vasudevan V, Reusche R, Wallace H, Kaza S. Clinical outcomes and cost-benefit analysis comparing laparoscopic and robotic colorectal surgeries. Surg Endosc 2016;30(12):5490-5493

39 Ke T-W, Geniales CR, Chen WT-L. The role of splenic flexure mobilization in laparoscopic rectal surgery for rectal cancer. Mini-invasive Surg 2018;2(10):35. Doi: 10.20517/25741225.2018 .46

40 Kye BH, Kim HJ, Kim HS, Kim JG, Cho HM. How much colonic redundancy could be obtained by splenic flexure mobilization in laparoscopic anterior or low anterior resection? Int J Med Sci 2014;11(09):857-862

41 Schlussel AT, Wiseman JT, Kelly JF, et al. Location is everything: The role of splenic flexure mobilization during colon resection for diverticulitis. Int J Surg 2017;40:124-129

42 Isik O, Sapci I, Aytac E, et al. Laparoscopy reduces iatrogenic splenic injuries during colorectal surgery. Tech Coloproctol 2018; 22(10):767-771

43 Isik O, Benlice C, Gorgun E. A novel approach for robotic mobilization of the splenic flexure. Tech Coloproctol 2017;21(01):53-57

44 Tocchi A, Mazzoni G, Fornasari V, Miccini M, Daddi G, Tagliacozzo S. Preservation of the inferior mesenteric artery in colorectal resection for complicated diverticular disease. Am J Surg 2001; 182(02):162-167 
45 Lehmann RK, Brounts LR, Johnson EK, Rizzo JA, Steele SR. Does sacrifice of the inferior mesenteric artery or superior rectal artery affect anastomotic leak following sigmoidectomy for diverticulitis? A retrospective review. Am J Surg 2011;201(05): 623-627

46 Cirocchi R, Trastulli S, Farinella E, et al. Is inferior mesenteric artery ligation during sigmoid colectomy for diverticular disease associated with increased anastomotic leakage? A meta-analysis of randomized and non-randomized clinical trials. Colorectal Dis 2012;14(09):e521-e529

47 Masoni L, Mari FS, Nigri G, c Preservation of the inferior mesenteric artery via laparoscopic sigmoid colectomy performed for diverticular disease: real benefit or technical challenge: a randomized controlled clinical trial. Surg Endosc 2013;27(01): 199-206

48 Dobrowolski S, Hać S, Kobiela J, Sledziński Z. Should we preserve the inferior mesenteric artery during sigmoid colectomy? Neurogastroenterol Motil 2009;21(12):1288-e123

49 Keller DS, Ishizawa T, Cohen R, et al. Elsevier Editorial System(tm) for The Lancet Gastroenterology \& Hepatology Manuscript Draft Indocyanine Green Fluorescence Imaging in Colorectal Surgery: Reviewing the Current Literature, Applications, and Future Direction. Lancet 2017http://discovery.ucl.ac.uk/10045273/1/THELANCETGASTROHEP-D-17-00224R1.pdf. Accessed July 15, 2020
50 Jafari MD, Wexner SD, Martz JE, et al. Perfusion assessment in laparoscopic left-sided/anterior resection (PILLAR II): a multiinstitutional study. J Am Coll Surg 2015;220(01):82-92.e1

51 Degett TH, Andersen HS, Gögenur I. Indocyanine green fluorescence angiography for intraoperative assessment of gastrointestinal anastomotic perfusion: a systematic review of clinical trials. Langenbecks Arch Surg 2016;401(06):767-775

52 Bae SU, Min BS, Kim NK. Robotic low ligation of the inferior mesenteric artery for rectal cancer using the firefly technique. Yonsei Med J 2015;56(04):1028-1035

53 Ryu S, Yoshida M, Hironori O, et al. Intraoperative ICG fluorescence contrast imaging of the main artery watershed area in colorectal cancer surgery: report of a case. Int J Surg Case Rep 2016;26:176-178

54 Sherwinter DA, Gallagher J, Donkar T. Intra-operative transanal near infrared imaging of colorectal anastomotic perfusion: a feasibility study. Colorectal Dis 2013;15(01):91-96

55 Blanco-Colino R, Espin-Basany E. Intraoperative use of ICG fluorescence imaging to reduce the risk of anastomotic leakage in colorectal surgery: a systematic review and meta-analysis. Tech Coloproctol 2018;22(01):15-23

56 Hellan M, Spinoglio G, Pigazzi A, Lagares-Garcia JA. The influence of fluorescence imaging on the location of bowel transection during robotic left-sided colorectal surgery. Surg Endosc 2014;28 (05):1695-1702 